able to carry out a straightforward psychiatric assessment and thereafter make sensible use of the available facilities.

But was the assessment by either the physicians or the psychiatrists at a satisfactory level ? It all depends on the definition of the word "relapse," which is used more than once but never defined. If this just means "psychiatric breakdown" in this context, then its usage is inherently vague but otherwise unremarkable. If it means a second suicide attempt it is alarming, since this would mean that $28.75 \%$ of the physicians' and $35 \%$ of the psychiatrists' patients had repeated within one year of the first attempt. This cannot be, since the authors say that there were "312 consecutive admissions (276 patients) to the trial," which would imply that 36 patients had repeated in a year, which is approximately $13^{\circ} \circ$ and a gratifyingly low proportion. Unfortunately a little later they mention that 24 patients were admitted more than once to the trial. Does this imply that some were admitted three or more times? It is irritating to go over data several times and to be unable to extract their significance because the authors have given us insufficient information.

Having worked in a poisons treatment centre in the past I remain impressed with the concept and agree with Kennedy ${ }^{2}$ and with the Department of Health that these are both useful and desirable. In my view it is in such centres that we can develop clinical, research, and didactic activities that will lead to a truly inter-disciplinary approach to the frightening epidemic of parasuicide. Ad-hoc (or more likely, ad-hominem) arrangements as described by Dr Gardner and his colleagues are worthy efforts but are imperilled by every rotation of junior staff and every new physician appointment.

Could the authors clarify their data and, in addition, tell us whether, in 1978, almost three years after their study, the system they describe is still working as well as they would like?

Alistair Munro

Department of Psychiatry,

Toronto General
Toronto, Ontario

1 Patel, A R, British Medical fournal, 1975, 2, 426.
2 Kennedy, P, British Medical fournal, 1972, 4, 255.

***We sent a copy of this letter to Dr Gardner and his colleagues, whose reply is printed below.-ED, BMF.

SIR,-Dr Munro has made a plea for the retention of the Department of Health's recommendation $^{1}$ (and its introduction to Toronto?) even though he is unable to cite any empirical evidence in its support 10 years after the recommendation was made. Yet he agrees with our findings and, in his letters, repeats several of our views precisely.

His main objection is to the general application of our findings to other hospitals because of the "adverse attitudes" prevailing there. If another hospital were to repeat our work and come to the same conclusion would that hospital also be atypical ? Perhaps such a prospective clinical trial would have to be carried out in every hospital. We anticipated this objection and tried to minimise it by arranging for all the physicians and psychiatrists who customarily treated self-poisoned patients admitted to this hospital to participate in the trial.

If instead of asking us to "clarify our data" he had read our paper carefully he would have found the answers to several of his questions. The definition of the word relapse is on $p$ 1568 and on the same page we explain about the reallocation of patients in the trial. It would also have been obvious to him that a few patients must have been admitted to the trial on more than two occasions. He clearly misunderstands the concept of the trial. The figures are higher because the follow-up was not confined only to those patients who were readmitted to Addenbrooke's and who attended the accident department but also included all patients who relapsed elsewhere. Taking this into account we think that our assessments a Addenbrooke's were at a "satisfactory level." The relapse rates were calculated using standard statistical techniques for follow-up studies-that is, the figures were corrected for patients who had been lost to follow-up for some reason. Had we taken the crude figures from Tables IV and I we would have obtained slightly different relapse rates of $22 \%$ and $25 \%$ for first admissions to the trial. More "gratifying" figures might have been obtained had we not taken the trouble of following up our patients about the country.

To answer Dr Munro's final question, the system described in our paper is not only working three years after the study but has been fully adopted since November 1977 so that the medical teams now make the initial psychiatric assessment and ask for advice from psychiatrists and social workers as necessary. We continue to place emphasis on the instruction of junior doctors and nurses, on the education of medical students, and on improving the treatment and after-care services.

R GARDNER R HANKA

Addenbrooke's Hospital

A J F PAGE Cambridge

'Central and Scottish Health Services Councils, Hospital Treatment of Acute Poisoning. London, HMSO, 1968.

\section{Delayed respiratory depression after use of fentanyl}

SIR, - The paper by Drs A P Adams and D A Pybus (4 February, p 278) raises important implications for the postoperative care of patients with the increasing use of high doses of analgesics, particularly fentanyl, during anaesthesia. However, in the discussion no mention is made of the fact that all their patients were less than average in weight and that all had received narcotic premedication, including hyoscine in two of the cases.

It is my observation that even small doses of morphine used as premedication can result in potentiated respiratory depression postoperatively, and for this reason patients undergoing major maxillofacial surgery are always given a non-narcotic premedication, usually oral diazepam and droperidol, the doses varying with the size of patient and degree of sedation required, and if necessary a sleeping state can be produced with nc evidence of respiratory depression. I use large $(0 \cdot 5-\mathrm{mg})$ doses of fentanyl and postoperative respiratory depression has never occurred while these patients are observed for the following $24 \mathrm{~h}$ in the intensive care unit.

With many alternative drugs available there seems little justification for using narcotic analgesics as premedication if there is any risk of further respiratory depression occurring during anaesthesia and presenting a postoperative hazard to the patient.

\section{Christopher J WRIGHT}

\section{St James's University Hospital,}

Leeds

SIR,-The paper by Drs A P Adams and D A Pybus (4 February, p 278) raises considerable doubts about the safe use of fentanyl in anaesthesia and reports three serious cases of delayed respiratory depression.

I believe that this respiratory depression is already clinically well recognised and is associated with the premedicant drugs mentioned-morphine and papaveretum-whose effects are potentiated by fentanyl. I regard papaveretum as the best narcotic premedican for many patients but have reluctantly abandoned it before neuroleptanaesthesia precisely because of the delayed respiratory depression. Naloxone is very effective in dealing with this, but, pari passu, abolishes the analgesia which is one of the main objects of the technique.

I teach now that premedication before medium-dose fentanyl neuroleptanaesthesia (0.5-1 $\mathrm{mg})$ should consist either of fentanyl itself in the form of Thalamonal (fentanyldroperidol mixture) or, failing this, a pethidine mixture. Personally I would not proceed to a "neurolept" sequence if morphine or papaveretum had been given in the last $12 \mathrm{~h}$.

Royal Sussex County Hospital,

J H Williams

Brighton

\section{The water story}

SIR,-The "water story" leading article (4 February, p 264) is certainly an interesting one. There is, nevertheless, a problem in comparing British and American studies of possible links between the softness of water and patterns of cardiovascular disease. ${ }^{1}$

The situation in American soft-water areas is simply not comparable with our own, at least where human calcium intake is concerned. Firstly, the definition of "soft" water differs, but, more important, Americans in soft-water areas have probably grown up consuming far more calcium than people nurtured on our chalkiest streams. The reason is that until the early ' 50 s milk consumption was substantially higher than in the UK and until the ' 40 s it was very much higher. This covers most of the lifetime of the majority of subjects in the cardiovascular studies. Since cows' milk contains about $1.25 \mathrm{~g} / 1$ of calcium even a difference of less than half a litre in daily milk consumption would have provided Americans with as much extra calcium as Britons drinking over a litre of the very hardest unboiled water (430 ppm calcium). Thus where British and American studies agree on an effect of soft water it seems unlikely that the common factor is a low basal intake of calcium.

Department of Physiology, Royal Veterinary College,

Alastair Micheli ${ }^{1}$ Michell, A, Perspectives in Biology and Medicine, 1976

SIR,-In your leading article on this subject (4 February, $p$ 264) you state that there is a lack of theoretical explanation for the inverse 